\title{
Crystal structure of SecB from Escherichia coli
}

\author{
Carien Dekker, ${ }^{\mathrm{a}, \mathrm{b}, \mathrm{c}, *}$ Ben de Kruijff, ${ }^{\mathrm{c}}$ and Piet Gros ${ }^{\mathrm{b}}$ \\ ${ }^{a}$ National Institute for Medical Research, The Ridgeway, Mill Hill, London, England, NW7 1AA, UK \\ b Department Crystal and Structural Chemistry, Utrecht University, Padualaan 8, NL-3584 CH Utrecht, The Netherlands \\ ${ }^{c}$ Department Biochemistry of Membranes, Utrecht University, Padualaan 8, NL-3584 CH Utrecht, The Netherlands
}

Received 11 July 2003, and in revised form 1 September 2003

\begin{abstract}
The chaperone SecB from Escherichia coli is primarily involved in passing precursor proteins into the Sec system via specific interactions with SecA. The crystal structure of SecB from E. coli has been solved to $2.35 \AA$ resolution. The structure shows flexibility in the crossover loop and the helix-connecting loop, regions that have been implicated to be part of the SecB substrate-binding site. Moreover conformational variability of Trp36 is observed as well as different loop conformations for the different monomers. Based on this, we speculate that SecB can regulate the access or extent of its hydrophobic substrate-binding site, by modulating the conformation of the crossover loop and the helix-connecting loop. The structure also clearly explains why the tetrameric equilibrium is shifted towards the dimeric state in the mutant SecBCys76Tyr. The buried cysteine residue is crucial for tight packing, and mutations are likely to disrupt the tetramer formation but not the dimer formation.
\end{abstract}

(C) 2003 Elsevier Inc. All rights reserved.

Keywords: SecB; Chaperone; Crystal structure; Protein translocation; Escherichia coli

\section{Introduction}

As protein synthesis in Escherichia coli takes place in the cytosol, export is essential for proteins functioning outside the cytosol. Early steps in this export process involve the translocation of precursor proteins across the inner membrane. This is accomplished by the translocation machinery, a well studied complex of Sec-proteins (for a review see (Driessen et al., 2001)). The integral membrane proteins SecY, SecE, and SecG form together with the peripherally bound ATPase SecA the translocase. SecA is able to insert and deinsert into the membrane upon binding and hydrolysis of ATP, providing the energy to drive the translocation process (Driessen et al., 1995). SecA has also been found in the cytosol and together with SecB it forms the soluble part of the translocation machinery (Hartl et al., 1990). SecB is a molecular chaperone that binds to a subset of precursor proteins, thereby preventing

\footnotetext{
${ }^{*}$ Corresponding author. Fax: +44-208-816-2580.

E-mail address: cdekker@nimr.mrc.ac.uk (C. Dekker).
}

them from aggregating (Kumamoto, 1990). The affinity of SecB for the C-terminus of SecA plays an important role in the correct targeting of the precursor protein to the translocase (Breukink et al., 1995). SecB is active as a tetramer and is highly selective towards its substrates. SecB substrates share no sequence homology, but a minimal binding motif consists of nine residues of charged and aromatic amino acids (Knoblauch et al., 1999). Although it is the signal sequence that is an earmark for export proteins, SecB does not exploit this as a binding motif but mainly binds to regions in the mature part of precursor proteins (Topping and Randall, 1994). Tight binding of SecB to a substrate is likely to be modulated by the ability of the chaperone to interact at multiple sites of a single substrate (Randall et al., 1998).

Previously, the crystal structure of SecB from Haemophilus Influenzae was determined to $2.5 \AA$ resolution (Xu et al., 2000). Here we report the three-dimensional crystal structure of SecB from E. coli at $2.35 \AA$ resolution. Together with a wealth of biochemical data that is available for this $E$. coli chaperone, the structure allows us to speculate on the mechanism by which 
SecB binds and releases precursor proteins and interacts with SecA.

\section{Materials and methods}

\subsection{Crystallisation and data collection}

SecB was crystallised as described previously (Dekker et al., 1999). Diffraction data were collected from a single crystal at EMBL beamline X11 (DESY, Hamburg). A $\mathrm{V}_{\text {matthews }}$ of $2.6 \mathrm{~A}^{3} / \mathrm{Da}$ suggests one tetramer per asymmetric unit, with a solvent content of $53.2 \%$. Data were processed with DENZO and scaled with SCALEPACK (Otwinowski and Minor, 1997). Table 1 summarises data collection and processing statistics.

\subsection{Structure solution and refinement}

The structure was solved by molecular replacement with AmoRe (Navaza, 1994), using as a search model the co-ordinates of residues 19-146 of $H$. Influenzae SecB (PDB entry code 1FX3)(Xu et al., 2000). A clear solution was obtained using data in the $20-4.0 \AA$ range. Cycles of positional and temperature factor refinement with CNS v.1.1 (Brunger et al., 1998), applying bulk solvent and global anisotropic B-factor corrections, alternating with manual model building using $\mathrm{O}$ version 7.0 (Jones et al., 1991), allowed us to improve the model and to place the E. coli $\mathrm{SecB}$ specific loops and side chains. Because of non-crystallographic symmetry (NCS) reflections in the test set were chosen in thin shells. The final model contains for monomer A, residues 9-142, for monomer B, residues 9-141, for monomer C, residues 9-140, for monomer D, residues 9-144 and 113 water molecules. All residues are found in allowed regions of the Ramachandran plot, as verified with PROCHECK (CCP4, 1994).

Atomic co-ordinates and structure factors are deposited in the PDB, entry code $1 \mathrm{QYN}$. Table 2 presents a summary of the refinement statistics.

Table 1

Data collection statistics

\begin{tabular}{ll}
\hline Resolution & $2.35 \AA$ \\
Space group & $\mathrm{P} 4_{3} 2_{1} 2$ \\
Unit cell & $\begin{array}{l}a=b=84.0 \AA \\
\\
\end{array}=204.8 \AA$ \\
& $\alpha=\beta=\gamma=90^{\circ}$ \\
Temperature & $100 \mathrm{~K}$ \\
No. of reflections & 465596 \\
No. of unique refl. & 33605 \\
$R_{\text {merge }}$ (highest res. shell) & $4.5 \%(21.9 \%)$ \\
$\langle I\rangle / \sigma|I\rangle$ (highest res. shell) & $28.6(5.0)$ \\
Completeness & $95.8 \%$ \\
\hline
\end{tabular}

Table 2

Refinement statistics

\begin{tabular}{ll}
$\begin{array}{l}\text { Molecular replacement } \\
\text { Model }\end{array}$ & $\begin{array}{l}\text { H. Influenzae tetramer residues 19-146 } \\
R \text {-factor (rot/trans) }\end{array}$ \\
$\begin{array}{l}\text { Refinement statistics } \\
\text { (CNS) }\end{array}$ \\
Resolution range & $29.6-2.35 \AA$ \\
No. of non-hydrogens & 4241 \\
No. of waters & 113 \\
$R$ (work) & $23.5 \%$ \\
$R$ (free) (10\%-thin shells) & $27.1 \%$ \\
rmsd bond lengths & $0.011 \AA$ \\
rmsd angles & $1.36^{\circ}$ \\
Average B & $41.3 \AA^{2}$ \\
Ramachandran & $88.1 \%$ core \\
& $11.9 \%$ allowed \\
& $0 \%$ generously allowed \\
& $0 \%$ disallowed \\
\hline
\end{tabular}

\section{Results and discussion}

\subsection{Structure description}

The crystal structure of SecB from E. coli was solved by molecular replacement using the co-ordinates of SecB from $H$. Influenzae (Xu et al., 2000), which has 55\% sequence identity with $\mathrm{SecB}$ from $E$. coli. Fig. 1 shows the $\mathrm{C} \alpha$ backbone of the chaperone. SecB is a 155 -residue protein that forms a tetramer with a molecular mass of $68.8 \mathrm{kDa}$. The tetramer is build up as a dimer of dimers. Each monomer named $\mathrm{A}$ to $\mathrm{D}$, folds into a 4-stranded Greek key anti-parallel $\beta$-sheet with a long $\alpha$-helix packed against the hydrophobic side of the sheet. Secondary structure elements are indicated in Fig. 1C. The dimer is formed by a $180^{\circ}$ rotation of monomer $\mathrm{B}$ with respect to monomer $\mathrm{A}$, so that strand $\beta 1(\mathrm{~A})$ lays antiparallel to strand $\beta 1(B)$ and the two sheets form one large 8 -stranded $\beta$-sheet. Two dimers establish a tetramer by packing their $\alpha 1$-helices in between the $\beta$-sheets. The model is not complete, as $8 \mathrm{~N}$-terminal residues and $11 \mathrm{C}$-terminal residues are not visible in the electron density maps. The direction of extension of the C-terminus is unclear, as for monomers $\mathrm{A}, \mathrm{B}$ and $\mathrm{C}$ an extended $\alpha$-helix free standing in solution is unlikely due to crystal contact sites. A possibility is that the extreme Cterminus is not visible, because it does not continue as a $\alpha$-helix but packs against the neighbouring tetramer as random coil. A similar degree of disorder at the $\mathrm{N}$ and $\mathrm{C}$ termini was observed in the case of $H$. Influenzae SecB, where residues $15-151$ on a total of 169 residues were incorporated in the model (Xu et al., 2000).

A superposition of the four monomers (Fig. 1C) reveals nearly identical conformations with a root mean square deviation of $C \alpha$-atoms between 0.39 and $0.75 \AA$, calculated pair wise. There are differences in the $\beta$-strand connecting loops and in the helix-connecting loop. 
The crossover loop, connecting strand $\beta 1$ and $\beta 2$, and the $\beta$-hairpin, connecting strand $\beta 3$ and $\beta 4$, of monomer $\mathrm{D}$ are involved in a crystal contact. The different conformations are likely to indicate flexibility for the hairpin and the crossover loop, as can also be concluded from the high B-factors in these regions. From a functional point of view, the flexibility may be essential in those regions as the cross-over loop and the helixconnecting loop have been implicated to be part of the SecB substrate binding site (Xu et al., 2000).

\subsection{Comparison with $H$. Influenzae $\operatorname{SecB}$}

At the amino acid sequence level the homology between $H$. Influenzae and E. coli $\mathrm{SecB}$ is high with $59 \%$ identity over a stretch of 132 residues. The total length of E. coli $\mathrm{SecB}$ is 155 residues, whereas $H$. Influenzae SecB comprises 169 residues. Apart from H. Influenzae SecB having additional residues at the very $\mathrm{N}$ - and $\mathrm{C}$-termini, there is only one insert of two residues in the loop connecting $\beta$-strands 3 and 4 . In fact, in the $E$. coli protein this loop is a two-residue $\beta$-hairpin (res. 69-70) that will probably be more restricted in mobility than the longer $H$. Influenzae loop. A superposition of $E$. coli SecB and $H$. Influenzae SecB (Xu et al., 2000) reveals the high similarity (data not shown). Differences between the two structures are localised in the loop regions and at the side chain level.

\subsection{Dimer of dimers}

Before structural data on SecB was available, biochemical data indicated that tetrameric SecB is a dimer of dimers (Topping et al., 2001). Fig. 2 shows in detail the residues involved in dimer stabilisation. The dimer is stabilised by main chain hydrogen bonding between strand $\beta 1$ from two monomers. Additionally, there are hydrogen bonds between side chains from the two $\alpha 1$ helices, and hydrogen bonds between helix $\alpha 1$ from one monomer and strand $\beta 1$ from the rotated monomer.

The dimer-dimer interface reveals hydrogen bonding between $\beta 1$ from one dimer and $\alpha 1$ from the other, as well as hydrogen bonding between the $\alpha 1$ helices. The hydrogen bonds at the dimer-dimer interface are outnumbered by the hydrogen bonds involved in dimer formation (respectively, 12 and 21 hydrogen bonds $<3.3 \AA$ ). Moreover, the tetramer is stabilised by hydrogen bonding via water molecules that are located at the dimer-dimer interface. These hydrogen contacts with solvent molecules are interesting when considering the fact that tetrameric SecB is in dynamic equilibrium with its dimeric form (Topping et al., 2001). The involvement of solvent molecules in the formation of the tetramer may facilitate a rapid exchange of dimers via the free solution.

It has been shown that the tetrameric state of $\mathrm{SecB}$ can be disrupted by certain mutations (Muren et al., 1999). Mutations Cys76Tyr, Val78Phe, and Gln80Arg shift the equilibrium towards the dimeric state. The side chains of these residues are located inside the molecule and are involved in interactions between the $\beta$-sheet and helix $\alpha 1$. Especially Cys76, which is the residue showing the most pronounced effect when mutated, is located on the inside of the $\beta$-sheet and is in close proximity of Met117 that is part of helix $\alpha 1$. In fact, there is an unusual 'sulphur-centre' visible where Cys76 is at van der Waals distance with Met117 and Cys113, whereas also Val78 and Ile21 are in close proximity (Fig. 3). In the electron density maps there is density for multiple conformations for both Cys76 and Met117 (Fig. 3B). As Met117 is part of helix $\alpha 1$, it is conceivable that a mutation of Cys76 into the more bulky tyrosine has a destabilising effect on the tetramer. In fact, when analysing a model of SecBCys76Tyr after energy minimisation, it shows that within one monomer the $\beta$-sheet and helix $\alpha 1$ are pushed apart. This is likely to have an effect on tetramerisation, more so than on dimerisation in which case interactions are mainly helix-to-helix and sheet-tosheet and are less likely to be affected by the tightness of the helix-to-sheet packing (Fig. 4). Although mutation Cys76Tyr may cause only a minor conformational change within one monomer, its destabilising effect on the tetramer by changing the dimer-dimer interface will shift the dynamic equilibrium towards the dimeric state, as revealed by various techniques (Kimsey et al., 1995).

\subsection{Substrate binding}

The two peptide binding subsites, as proposed by $\mathrm{Xu}$ et al. (Xu et al., 2000), meet all the requirements for substrate binding by SecB. The aromatic, deep subsite 1 and the hydrophobic, shallow subsite 2 together form an extended groove that could easily accommodate the SecB binding motif, being a stretch of about 10 residues with preferably aromatic residues and at least 3 basic residues (Knoblauch et al., 1999). In fact, if the ligand were in an extended conformation, up to 20 residues would fit in the $70 \AA$ long binding groove on either side of the molecule. Fig. 5 shows the location of the subsites. Charged residues near subsite 2, like Asp43 and Asp45 have been postulated to act as a selectivity filter for ligands (Randall and Hardy, 2000). A surface potential plot, as shown in Fig. 5, shows that E. coli $\mathrm{SecB}$ is a highly negatively charged molecule. Whereas SecB is negatively charged at and near subsite 2 , it shows patches of a more neutral and slightly positively charged surface near subsite 1 . This is fully consistent with the hypothesis by Randall (Randall and Hardy, 1995) that $\mathrm{SecB}$ binds it substrates initially via electrostatic interactions whereas hydrophobic interactions subsequently account for a tight complex. As suggested before (Randall and Hardy, 1995), SecB is able to interact at least transiently with many more proteins than just the SecBdependent ones, but only the precursors containing the 

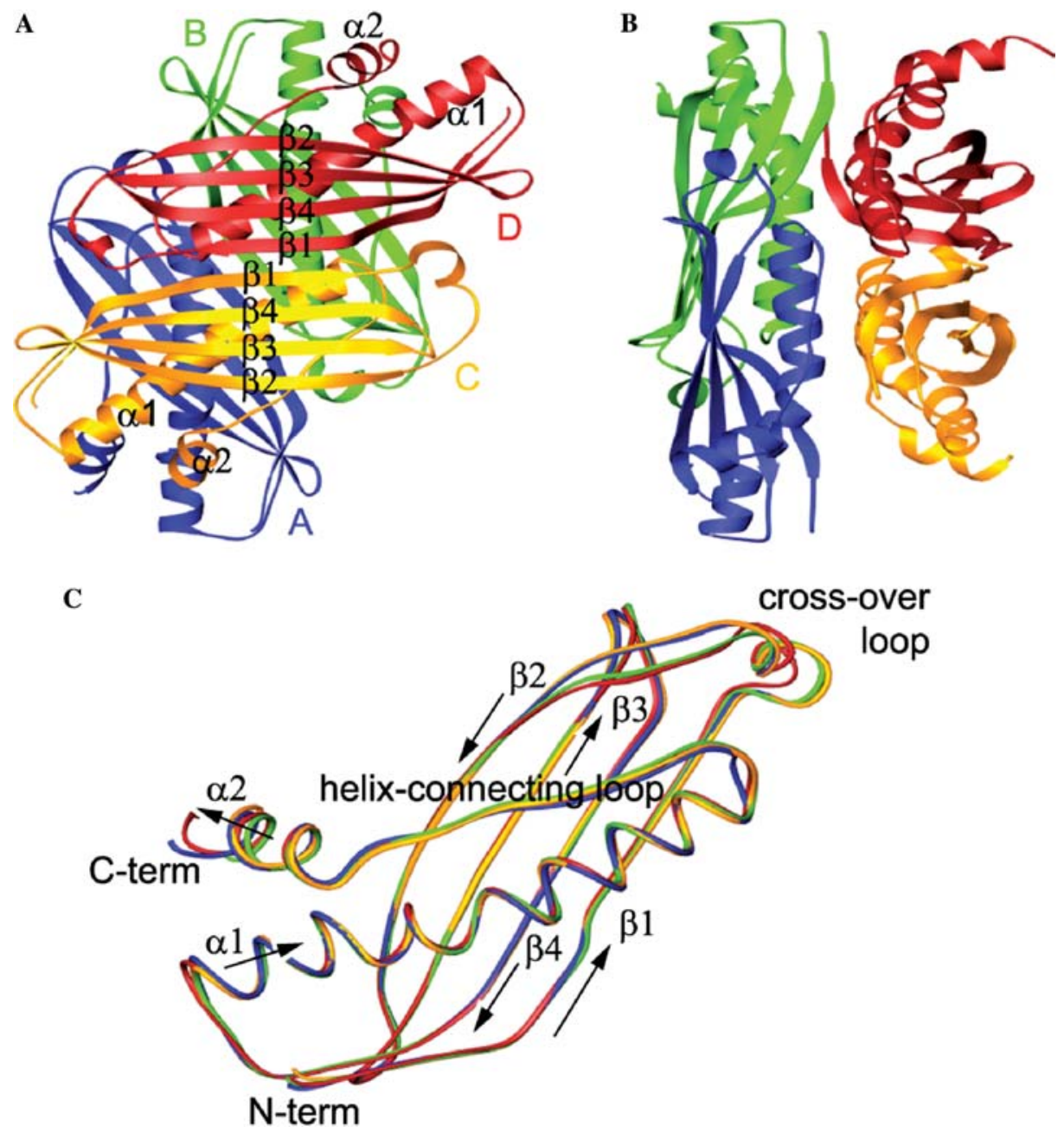

Fig. 1. Overall structure. Overall structure of tetrameric SecB from E. coli. (A) Front view, showing the 8-stranded $\beta$-sheet formed by two monomers. (B) Side view, showing the dimer-dimer interface formed by the $\alpha$-helices. (C) Superposition of the four monomers revealing variability in the loop region connecting strand $\beta 3$ and $\beta 4$, and in the crossover loop. Also indicated are the main secondary structure elements, and the $\mathrm{N}$ - and $\mathrm{C}$-terminus. Figs. 1-3 were created using Molscript (Kraulis, 1991).

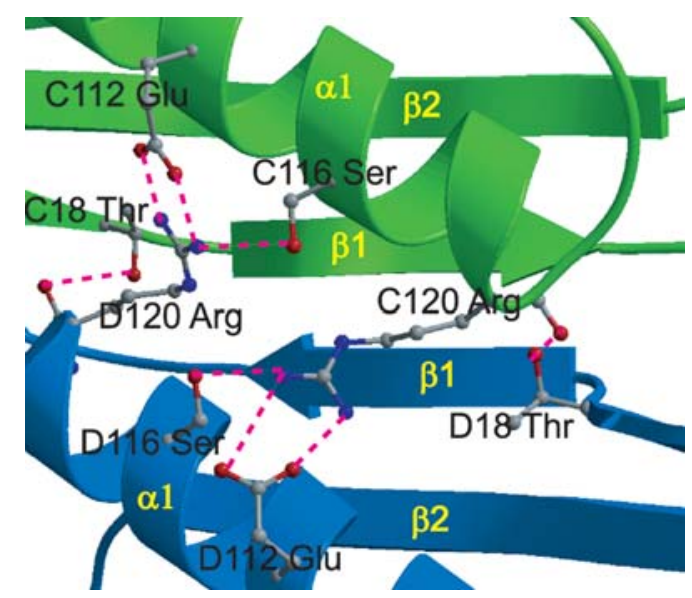

Fig. 2. Dimer interface. Detail of hydrogen bonding involved in the dimer interface. Monomer $\mathrm{C}$ is shown in green, monomer $\mathrm{D}$ is shown in blue. Hydrogen bonds are represented by magenta dotted lines. Main chain hydrogen bonds between strands $\beta 1(\mathrm{C})$ and $\beta 1(\mathrm{D})$ are not indicated. right binding motif will form a stable complex with the chaperone.

In monomers A and C, Trp36, which is located within subsite 1, points inwards and is tucked away inside the protein. In monomers $\mathrm{B}$ and $\mathrm{D}$, however, this residue is much more exposed, partly because of a crystal contact. This difference in conformation may illustrate how $\mathrm{SecB}$, by using the flexibility in the loop regions, can change its hydrophobic surface. A change in hydrophobic surface as a consequence of peptide binding has been observed (Randall and Hardy, 1995). The crystal structure enables us to speculate on the structural basis of such an effect. Based on the observed conformational variability of Trp36, and taking into account the different loop conformations as observed for the different monomers, we speculate that $\mathrm{SecB}$ can regulate the access or extent of its hydrophobic subsite 1, by modulating the conformation of the crossover loop and the helix-connecting loop. 
A

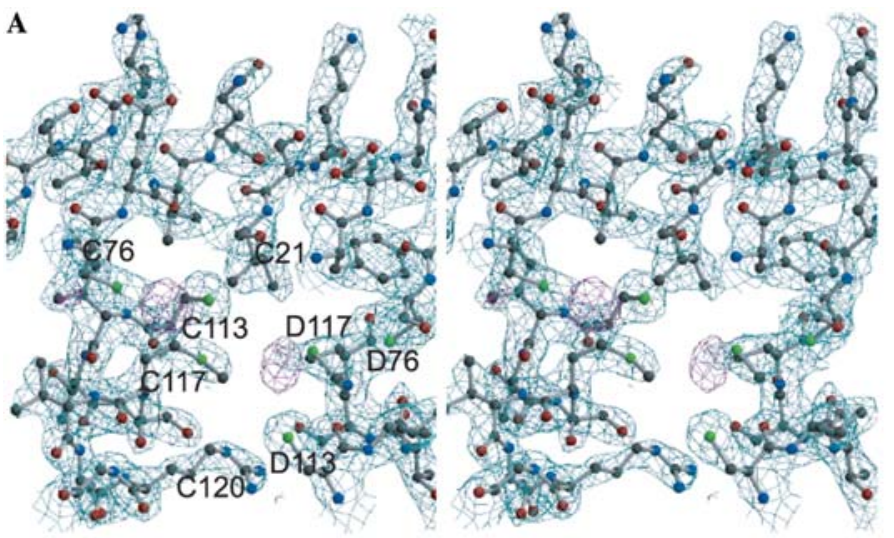

B

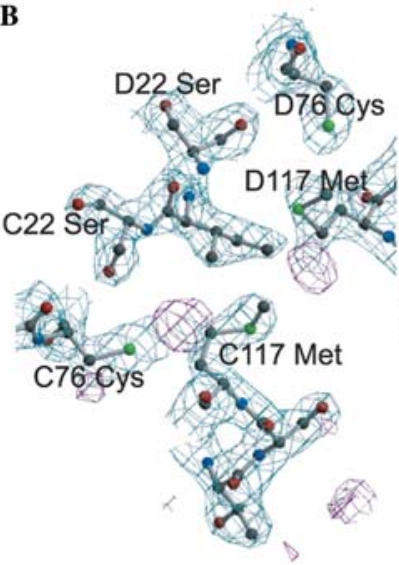

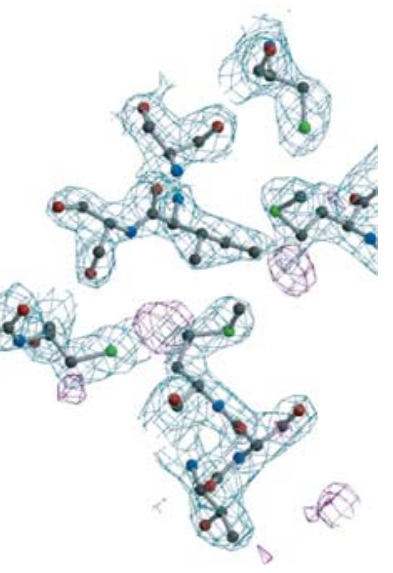

Fig. 3. Electron density of sulphur atoms. (A) Stereo picture showing the close proximity of sulphur atoms at the dimer interface. In blue the (2Fo$\mathrm{Fc}$ ) density map plotted at $1.5 \sigma$ level, in magenta the $(\mathrm{Fo}-\mathrm{Fc})$ difference density at $3 \sigma$. Sulphur atoms are shown in green. (B) A close up showing how the $(\mathrm{Fo}-\mathrm{Fc})$ density indicates multiple conformations for Cys C76, Met C117, and Met D117. There is continuous density in the (2Fo-Fc) map running from residue $\mathrm{C} 76$ to residue $\mathrm{C} 117$.

It has been shown that the extreme C-terminus of SecB is rather flexible (Volkert et al., 1999). Also, the protease accessibility of this $\mathrm{C}$-terminus is affected by
A

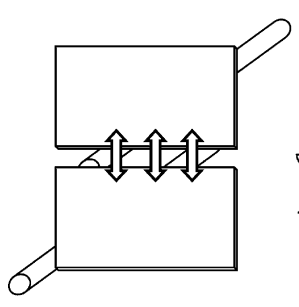

B

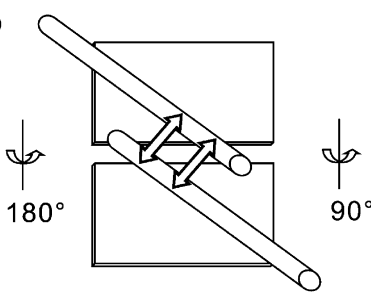

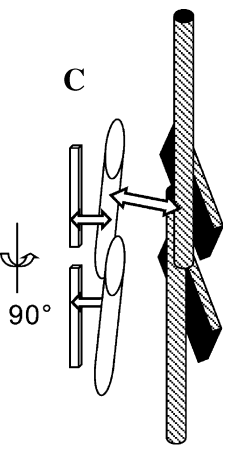

Fig. 4. Dimer-dimer model Schematic representation of a dimer of SecB. The $\beta$-sheet is represented by a rectangle, the $\alpha$-helix by a rod. The dimer interface is established by sheet-to-sheet interactions (A), and helix-to-helix interactions (B). Variability in the helix-to-sheet packing $(C)$ may not affect the dimer formation but will have an effect on the tetramer stability (the opposite dimer is shown in dashed lines). substrate binding (Randall, 1992). As the C-terminus is not visible in the electron density, we cannot speculate on its function, but it should not be ruled out that the $\mathrm{C}$-terminus might play a role in substrate binding. If the flexible $\mathrm{C}$-terminus is able to fold back onto substrate bound to $\mathrm{SecB}$, as speculated by Randall (Randall and Hardy, 2000), it may explain the fact that protease treatment of SecB-substrate complexes reveal protected fragments of 150 residues (Khisty et al., 1995). That is much more than the $\sim 20$ residues that would fit in the $70 \AA$ long binding grooves on either side of the chaperone. It therefore seems conceivable that the $\mathrm{C}$-terminus is somehow involved in substrate binding, and that its structural flexibility is related to that.

\subsection{SecA binding site}

The C-terminus of SecB has also been implicated in binding SecA, the part of the translocase to which $\operatorname{Sec} B$
A

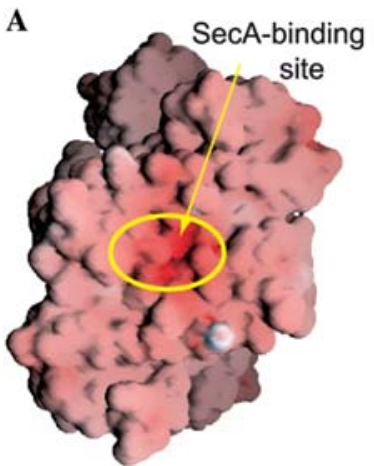

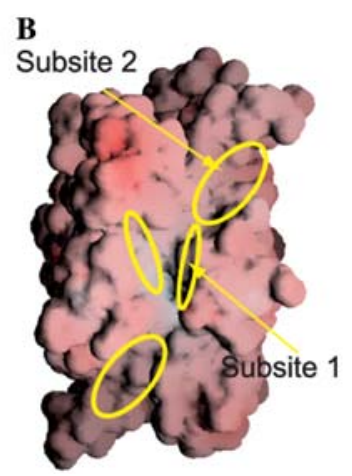

Fig. 5. Surface potential plot. Surface potential plot of tetrameric SecB, generated by GRASP (Nicholls et al., 1991) (electrostatic potential ranging from $-20 \mathrm{kT}$ (red) to $+20 \mathrm{kT}$ (blue)). (A) Front view, same orientation as Fig. 1A, facing the 8-stranded $\beta$-sheet, accommodating the SecA binding site. (B) Side view, showing the proposed peptide-binding groove harbouring subsite 1 and 2. (C) Same orientation as panel B, with hydrophobic residues in yellow, showing the shallow subsite 2 and the deeper subsite 1 . 
targets its substrates. It is the extreme C-terminus of SecA that binds to SecB (Fekkes et al., 1997). As mutational studies clearly indicate the involvement of Asp20, Glu24, Leu75, and Glu77 in SecA binding (Fekkes et al., 1998; Kimsey et al., 1995), the SecA binding site is well localised at the solvent exposed side of the $\beta$-sheet, right at the dimer interface (Driessen et al., 2001; Xu et al., 2000). The SecA binding site is a highly negatively charged region, as illustrated in Fig. 5A, and this will increase the affinity for the positively charged C-terminus of SecA. The SecA binding site is at the solvent exposed side of the central part of the $\beta$-sheet. The opposite side of this part of the sheet, facing the inside of the molecule, harbours the residues that are highly important for tetramer stabilisation (residues Cys76, Val78 and Gln80). Moreover, as shown in Fig. 3, these include residues that can adopt multiple conformations. It is therefore possible that binding of SecA to the exposed side of the $\beta$-sheet affects the residues involved in tetramerisation. This in turn may lead to changes in the conformation. Though not as drastic as dimerisation, minor changes at the dimer-dimer interface could contribute to the tight interplay between SecA and substrate-bound SecB. A similar SecA-SecB interaction model had been proposed before (Fekkes et al., 1998; Kimsey et al., 1995), in which binding of SecA to the hydrophilic side of the $\beta$-sheet was thought to directly influence the substrate binding site at the hydrophobic side of the $\beta$-sheet. However, the crystal structure of SecB reveals that the substrate binding site is remote from the SecA binding site. This suggests that the mechanism by which SecA modulates substrate binding on $\mathrm{SecB}$ is indirect and is likely to involve a conformational change. The $\mathrm{C}$-terminus of SecB has not been taken into account in this model but is likely to play a role in SecA binding. The recently published SecA structure (Hunt et al., 2002) does not contain the Cterminal SecB binding residues as they are likely to be disordered in the crystal. Therefore, we have to await the structure of SecA and SecB complexes before the intriguing fine-tuned interplay between these two proteins will be revealed.

\section{Conclusion}

The chaperone $\operatorname{Sec} \mathrm{B}$ from $E$. coli is primarily involved in passing precursor proteins into the Sec system via specific interactions with $\mathrm{Sec} A$. SecB is involved in two fundamentally different types of interactions: interactions with a diverse set of unfolded proteins, that are in rapid equilibrium with the free pool (Topping and Randall, 1997) and the unique interaction with SecA that makes the precursor enter the translocation pathway. SecB might also engage in functions completely unrelated to export (Panse et al., 2000) possibly acting as a general buffer for the unfolded state of polypeptides in the cytosol (Randall and Hardy, 2002).

The crystal structure of SecB from E. coli, as described in this paper, points out that the hypothesised substrate binding subsites are confined by flexible loop regions, suggesting that $\mathrm{Sec} B$ uses conformational flexibility to accommodate a wide range of substrates. We also observe conformational variability of Trp36, and different loop conformations for the different monomers. Based on this, we speculate that $\mathrm{Sec} B$ can regulate the access or extent of its hydrophobic subsite 1 , by modulating the conformation of the crossover loop and the helix-connecting loop. It is conceivable that binding of substrate to SecB leads to a conformational change, possibly leading to an increased exposed hydrophobic surface. The interplay between exposed hydrophilic sites and 'hidden' hydrophobic sites has been proposed earlier to be SecB's solution to the problem of combining high selectivity with low specificity (Randall and Hardy, 1995).

\section{Acknowledgments}

We are grateful to Clasien Oomen for her contribution. We thank V.S. Lamzin and M. Wilmanns for data collection at the EMBL at DESY, Hamburg. Support was provided under the EU TMR program to the EMBL Hamburg Outstation, Reference ERB4062PL970104. This work was supported in part by the Council of Chemical Sciences of the Netherlands Organisation for Scientific Research (NWO-CW). CD received support from the Commission of the European Communities (Grant QLK2-CT-1999-01293).

\section{References}

Breukink, E.J., Nouwen, N., van Raalte, A., Mizushima, S., Tommassen, J., de Kruijff, B., 1995. The C terminus of SecA is involved in both lipid binding and SecB binding. J. Biol. Chem. 270, 79027907.

Brunger, A.T., Adams, P.D., Clore, G.M., DeLano, W.L., Gros, P., Grosse Kunstleve, R.W., Jiang, J.S., Kuszewski, J., Nilges, M., Pannu, N.S., Read, R.J., Rice, L.M., Simonson, T., Warren, G.L., 1998. Crystallography \& NMR system: a new software suite for macromolecular structure determination. Acta Crystallogr. D 54, 905-921.

CCP4. 1994. Collaborative Computational Project No. 4: The CCP4 suite: programs for protein crystallography. Acta Crystallogr. D 50, 760-763

Dekker, C., de Kruijff, B., de Korte-Kool, G., Kroon, J., Gros, P., 1999. Crystals of acetylated SecB diffract to $2.3-\AA$ A resolution. J. Struct. Biol. 128, 237-242.

Driessen, A.J.M., de Wit, J.G., Kuiper, W., van der Wolk, J.P., Fekkes, P., van der Does, C., van Wely, K., Manting, E., den Blaauwen, T., 1995. SecA, a novel ATPase that converts chemical energy into a mechanical force to drive precursor protein translocation. Biochem. Soc. Trans. 23, 981-985. 
Driessen, A.J.M., Manting, E.H., van der Does, C., 2001. The structural basis of protein targeting and translocation in bacteria. Nat. Struct. Biol. 8, 492-498.

Fekkes, P., de Wit, J.G., van der Wolk, J.P.W., Kimsey, H.H., Kumamoto, C.A., Driessen, A.J.M., 1998. Preprotein transfer to the Escherichia coli translocase requires the co-operative binding of SecB and the signal sequence to SecA. Mol. Microbiol. 29, 11791190.

Fekkes, P., van der Does, C., Driessen, A.J.M., 1997. The molecular chaperone SecB is released from the carboxy-terminus of SecA during initiation of precursor protein translocation. EMBO J. 16, 6105-6113.

Hartl, F.-U., Lecker, S., Schiebel, E., Hendrick, J.P., Wickner, W., 1990. The binding cascade of SecB to SecA to SecY/E mediates preprotein targeting to the $E$. coli plasma membrane. Cell 63, 269-279.

Hunt, J.F., Weinkauf, S., Henry, L., Fak, J.J., McNicholas, P., Oliver, D.B., Deisenhofer, J., 2002. Nucleotide control of interdomain interactions in the conformational reaction cycle of SecA. Science 297, 2018-2026.

Jones, T.A., Zou, J.-Y., Cowan, J.W., Kjeldgaard, M., 1991. Improved methods for building protein models in electron density maps and the location of errors in these models. Acta Crystallogr. A 47, 110-119.

Khisty, V.J., Munske, G.R., Randall, L.L., 1995. Mapping of the binding frame for the chaperone SecB within a natural ligand, galactose-binding protein. J. Biol. Chem. 270, 25920-25927.

Kimsey, H.H., Dagarag, M.D., Kumamoto, C.A., 1995. Diverse effect of mutation on the activity of the Escherichia coli export chaperone SecB. J. Biol. Chem. 270, 22831-22835.

Knoblauch, N.T.M., Ruediger, S., Schoenfeld, H.J., Driessen, A.J.M., Schneider-Mergener, J., Bukau, B., 1999. Substrate specificity of the SecB chaperone. J. Biol. Chem. 274, 34219-34225.

Kraulis, P.J., 1991. MOLSCRIPT: a program to produce both detailed and schematic plots of protein structures. J. Appl. Crystallogr. 24, 946-950.

Kumamoto, C., 1990. SecB protein: a cytosolic export factor that associates with nascent exported proteins. J. Bioenerg. Biomem. 22, 337-351.

Muren, E.M., Suciu, D., Topping, T.B., Kumamoto, C.A., Randall, L.L., 1999. Mutational alterations in the homotetrameric chaperone SecB that implicate the structure as dimer of dimers. J. Biol Chem. 274, 19397-19402.
Navaza, J., 1994. AMoRe: an automated package for molecular replacement. Acta Crystallogr. A 50, 157-163.

Nicholls, A., Sharp, K.A., Honig, B., 1991. Protein folding and association: insights from the interfacial and thermodynamic properties of hydrocarbons. Proteins: Struct. Funct. Genet. 11, 281-296.

Otwinowski, Z., Minor, W., 1997. Processing of X-ray diffraction data collected in oscillation mode. Methods Enzymol. 276, 307-326.

Panse, V.G., Vogel, P., Trommer, W.E., Varadarajan, R., 2000. A thermodynamic coupling mechanism for the disaggregation of a model peptide substrate by chaperone SecB. J. Biol. Chem. 275, 18698-18703.

Randall, L.L., 1992. Peptide binding by chaperone SecB: implications for recognition of nonnative structure. Science 257, 241-245.

Randall, L.L., Hardy, S., Topping, T.B., Smith, V.F., Bruce, J.E., Smith, R.D., 1998. The interaction between the chaperone SecB and its ligands: evidence for multiple subsites for binding. Protein Sci. 7, 2384-2390.

Randall, L.L., Hardy, S.J., 2000. The promiscuous and specific sides of SecB. Nat. Struct. Biol. 7, 1077-1079.

Randall, L.L., Hardy, S.J., 2002. SecB, one small chaperone in the complex milieu of the cell. Cell. Mol. Life Sci. 59, 1617-1623.

Randall, L.L., Hardy, S.J.S., 1995. High selectivity with low specificity: how SecB has solved the paradox of chaperone binding. TIBS 20, 65-69.

Topping, T., Randall, L.L., 1997. Chaperone SecB from Escherichia coli mediates kinetic partitioning via a dynamic equilibrium with its ligands. J. Biol. Chem. 272, 19314-19318.

Topping, T.B., Randall, L.L., 1994. Determination of the binding frame within a physiological ligand for the chaperone SecB. Protein Sci. 3, 730-736.

Topping, T.B., Woodbury, R.L., Diamond, D.L., Hardy, S.J., Randall, L.L., 2001. Direct demonstration that homotetrameric chaperone SecB undergoes a dynamic dimer-tetramer equilibrium. J. Biol. Chem. 276, 7437-7441

Volkert, T.L., Baleja, J.D., Kumamoto, C.A., 1999. A highly mobile C-terminal tail of the Escherichia coli protein export chaperone SecB. Biochem. Biophys. Res. Commun. 264, 949-954.

$\mathrm{Xu}, \mathrm{Z}$., Knafels, J.D., Yoshino, K., 2000. Crystal structure of the bacterial protein export chaperone SecB. Nat. Struct. Biol. 7, 1172 1177 . 